\title{
Use of GeneXpert Assay for Diagnosis of Tuberculosis From Body Fluid Specimens, a 2 Years Study
}

\author{
Pravin $\mathrm{KN}^{1 *}$ and Chourasia $\mathrm{E}^{2}$ \\ ${ }^{1}$ Consultant Microbiologist \& Head Infection prevention, Holy Spirit Hospital, India \\ ${ }^{2}$ Consultant Microbiologist, Singapore
}

*Corresponding author: Pravin K Nair, Consultant Microbiologist \& Head Infection

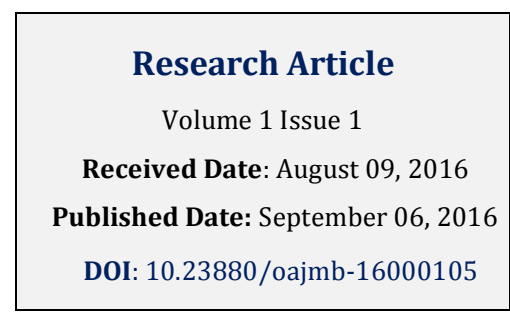
prevention, Holy Spirit Hospital, Mumbai, Maharashtra, India, Tel: +919833305875; Email: drpravinnair@gmail.com

\section{Abstract}

Tuberculosis (TB) remains a major health concern worldwide and India accounts for $23 \%$ of the global TB burden. Of all TB cases, extra pulmonary (EP) TB in India accounts for 15 to $20 \%$ that remains undetected and untreated not only due to its diverse clinical presentation but also due to lack of diagnostic means. EPTB poses a greater diagnostic challenge in HIV co-infected patients and children. Also with some forms of EPTB like TB meningitis, consequences can be lifethreatening. Early detection of TB and multidrug resistance is a priority in the diagnosis of TB for early disease management. In 2013, the World Health Organization has endorsed the use of GeneXpert MTB/RIF assay, a fullyautomated cartridge based nucleic acid amplification test for the rapid diagnosis of EPTB. We aimed to use the GeneXpert assay on body fluid specimens from patients with strong clinical suspicion, were hospitalized and the microscopic examination was negative for Acid-fast bacilli. GeneXpert assay was performed on 378 body fluid samples that consisted of 164 pleural fluids, $148 \mathrm{CSF}, 59$ ascitic fluid and 7 other body fluid (synovial, pericardial, peritoneal) specimens. Of these, 32 specimens were positive for MTB with a positivity rate of $10.1 \%, 8.8 \%$ and $9.3 \%$ respectively for pleural fluids, CSF and ascitic fluid. Rifampicin resistance was detected in 4 of the pleural fluid and one of the ascitic fluid samples. Collectively, $19.2 \%$ of the MTB positives in our study were multidrug resistant (MDR) TB. Thus the results suggest that GeneXpert assay has a good potential in the rapid accurate diagnosis of EPTB especially in body fluids, aids in prompt initiation of appropriate therapy and improving the quality of TB care.

Keywords: Extrapulmonary; Tuberculosis; Body Fluids; Nucleic-Acid Amplification; GeneXpert MTB/RIF

\section{Introduction}

Tuberculosis (TB) is a leading cause of death worldwide alongside HIV. According to WHO estimates for 2014, 1.5 million of TB deaths occurred globally. India, one of the nine high burden countries for TB, accounts for $23 \%$ (2.2 million) of the global TB burden, and for a third of the 'missing cases' that do not get diagnosed or notified
[1]. TB disproportionately involves the productive age group in India. Nearly $50 \%$ of multi-drug resistant TB cases are estimated to occur in China and India.

TB manifests clinically as pulmonary or extra pulmonary tuberculosis (EPTB), with the former being more common. It is estimated that 15 to $20 \%$ of all TB cases are extra pulmonary mainly affecting the lymph 


\section{Open Access Journal of Microbiology \& Biotechnology}

nodes, meninges, kidney, spine and growing ends of the bones [2]. With the advent of HIV infection, cases of EPTB have become more common. In such a scenario, rapid detection of TB cases and early determination of MDR-TB is highly important. A major hindrance to the diagnosis of EPTB is the atypical clinical presentation, often simulating neoplasia and/or inflammatory disorders. The diagnosis is further compromised by the paucibacillary nature of the disease or by extremely low bacterial load in nonrespiratory specimens [3].

Culture is the "gold standard" for detection of Mycobacterium tuberculosis (MTB) but it is slow and may take up to 2 to 8 weeks. Although microscopic examination for acid-fast bacilli (AFB) is rapid and inexpensive, it has poor sensitivity and specificity and is unable to differentiate between tuberculous and nontuberculous mycobacteria (NTM) [4]. Histological examination has its limitations as it cannot differentiate between TB and other related diseases like sarcoidosis or NTM infections except for the determination of stained tubercle bacilli. Several other tests that are also being employed for the diagnosis of EPTB include serological assays (both antigen and antibody detection), Mantoux test, and PCR assays (conventional as well as Real-time PCR); however, the specificities and sensitivities of these tests are variable. Also, these tests require a number of manual steps, and some have a relatively long turnaround time [5]. Thus, rapid identification is essential for early treatment initiation, improved patient outcome as well as for more effective public health intervention and it relies mainly on Nucleic acid amplification techniques (NAAT) [6]. Though other tests such a Line Probe assay (Hains life science) has been approved by WHO, but their main limitation is poor sensitivity in smear negative cases and being labor intensive.

Recently introduced GeneXpert MTB/RIF (Cepheid, Sunnyvale, CA, USA) assay, which can detect Mycobacterium tuberculosis complex and RIF resistance directly from clinical samples has been shown to be rapid, yielding results in less than 2 hours. The assay based on nested real-time PCR and molecular beacon technology is not prone to cross-contamination, requires minimal biosafety facilities, can be performed by technicians with little training; and has high sensitivity in smear-negative pulmonary TB (particularly relevant in HIV positive individuals) [7-11]. Based on an updated Cochrane systematic review, GeneXpert MTB/RIF (hence forth referred to as GeneXpert) assay has an overall sensitivity of $88 \%$ and a pooled specificity of $98 \%$ as compared to culture [12]. These characteristics make it a potentially useful technique in the diagnosis of EPTB as well. The information provided by GeneXpert assay helps in selecting treatment regimens and reaching infection control decisions quickly.

The study aimed at determining the utility of GeneXpert assay in the rapid diagnosis of EPTB in body fluids of patients with strong clinical suspicion of TB and with negative microscopic examination of ZN (ZiehlNeelsen) stained smears for Acid fast bacilli. Also to compare and contrast our results with similar studies.

\section{Materials and Methods}

A study was conducted in a private tertiary care hospital in Mumbai, India on clinical specimens collected over a period of 2 years from January 2014 to December 2015. Three hundred and seventy eight $(n=378)$ clinical samples were included in the study, all of which were collected from hospitalized patients. Based on the suspected site of localization, specimens were collected in appropriate quantity in sterile containers and sent to the Microbiology laboratory. These were pleural fluids $(n=164)$, CSF $(n=148)$, ascitic fluid $(n=59)$, pericardial fluid $(n=3)$, peritoneal fluid $(n=2)$ and synovial fluid $(n=2)$ specimens. This was entirely a laboratory based study and no other parameters were recorded other than those provided by the requesting clinician.

All the samples collected from clinically ill hospitalized patients were negative for acid fast bacilli by ZiehlNeelsen method, and culture results would have taken a much longer time to confirm the clinical suspicion. All this necessitated the need to test the body fluid specimens by GeneXpert assay.

\section{GeneXpert MTB/ RIF Assay}

GeneXpert assay, an automated molecular test for Mycobacterium tuberculosis (MTB) and resistance to rifampin (RIF), uses heminested real-time polymerasechain-reaction (PCR) assay to amplify an MTB-specific sequence (192 bp segment) of the rpoB gene, which is probed with molecular beacons for mutations within the rifampin-resistance determining region $[7,9]$.

\section{Sample Processing and Testing}

All the body-fluid samples, being collected from sterile sites did not receive any pre-treatment except for mechanical homogenization (if needed) [13]. Sample reagent (SR) was added to the sample at a ratio of 2:1, 


\section{Open Access Journal of Microbiology \& Biotechnology}

except for CSF (usually $<1 \mathrm{ml}$ ), which was raised to $2 \mathrm{ml}$ by the addition of SR buffer. The treated sample was incubated at room temperature for $15 \mathrm{~min}$ and then transferred to the test cartridge which is loaded into the GeneXpert instrument. The results obtained were interpreted as follows [11]:

- MTB detected; RIF resistance detected (MTB+, RIF+)

- MTB detected; RIF resistance not detected (MTB+, RIF-)

- MTB detected; RIF resistance indeterminate (MTB+, RIF In)

- MTB not detected (MTB-)

\section{Results and Discussion}

A total of 378 samples of body fluids were received in the Microbiology Laboratory for performing GeneXpert assay within 19 months period and were categorized as listed in. Pleural fluid accounted for $43.4 \%$ (164) of fluid samples, followed by $39.1 \%$ (148) accounted for by CSF and ascitic fluid accounted for $15.6 \%$ (59). The remaining comprised of pericardial $0.8 \%$ (3), peritoneal $0.5 \%$ (2) and synovial fluids $0.5 \%(2)$.

Globally the use of GeneXpert assay has resulted in an increase in the number of positive results by $16.5 \%$ and this increase has been more important for the extrapulmonary specimens especially the body fluids [14]. In our study, out of these 378 smear-negative body fluid specimens, 32 were positive for MTB of which 5 specimens also exhibited resistance to RIF. This indicates $19.2 \%$ of MTB positives were also multi-drug resistant (MDR-TB).

For Pleural fluid $10.1 \%$ was the Positive percentage (149 Negative, 4 Showed Rifampicin Resistance, 11 were Rifampicin sensitive). In Ascitic fluid we got a Positive percentage of $9.3 \%$ (54 Negative, 1 Rifampicin Resistance, 4 were sensitive).

The result for body fluids is comparable to the study done by Stephen D Lawn in 2012 [15]. Where the positivity rate in CSF was $5 \%$ as against $8.8 \%$ in our study. In a study from India [14], the positivity rate with GeneXpert assay was $24.6 \%$ for CSF, 32\% for pleural fluid and $27.8 \%$ for ascitic fluid. Another study from Pakistan have reported $15.8 \%, 40.0 \%$ and $6.3 \%$ respectively, of pleural fluid, CSF and ascitic fluid samples to be positive [16]. In various published studies, the GeneXpert sensitivities have ranged from $25.0 \%$ to $95.1 \%$ for EPTB specimens.
Hillemann et al found 3 positive GeneXpert results out of 113 pleural fluid samples (2.9\%) all of which were negative on mycobacterial culture, whereas the positivity rate for pleural fluids in our study was reported to be $10.1 \%$ [17]. Overall $9.4 \%$ of body fluids were tested positive in this study and is comparable to the results of Causes et al who reported 4 positive GeneXpert results out of 34 tested fluids (11.8\%) [18].

Data from prior studies suggested a potential role for nucleic acid amplification tests in the diagnosis of TB from CSF and the results for GeneXpert here are in accordance with the findings. It's been observed that GeneXpert assay does not reach the sensitivity of culture, however it can significantly improve/ fasten the diagnosis of CSF TB especially in places where expertise for culture or other diagnostic tests are not available or where a rapid diagnosis of TB is necessary (as in the case of tuberculous meningitis). While the detection time for MTB with GeneXpert assay was less than 2 hours in our present study, the mean turnaround time for culture positivity by Iqbal and Siddiqui has been reported to be 24 days and 23.13 days respectively in different studies $[19,20]$.

Although GeneXpert assay is considered a breakthrough in the diagnosis of TB and EPTB, one of the major limitations of this technique is that it cannot distinguish between viable and non-viable microorganisms while detecting MTB DNA. Hence it should not be used to monitor patients or efficacy of the treatment.

A major limitation of this study is not having comparative data with Culture.

This molecular technique of GeneXpert assay is relatively more expensive than traditional culture methods, however it makes an important contribution to the modern day detection of TB as it has higher sensitivity than smear and provides a more rapid diagnosis than culture and histology. Our findings support the routine use of GeneXpert assay for the diagnosis of EPTB in body fluids as time factor has a very crucial role in its laboratory diagnosis. To conclude, GeneXpert assay has the potential to significantly improve and escalate the diagnosis of smear-negative body fluid specimens at both hospital as well as point-of-care settings in regions not only with high TB burden but also with overlapping HIV. Also detection of RIF resistance aids in prompt initiation of appropriate therapy and thus improving the overall quality of TB care. 


\section{Open Access Journal of Microbiology \& Biotechnology}

\section{References}

1. (2015) World Health Organization: Global tuberculosis report.

2. Annual report - TB India (2015) Central TB division, Directorate General of Health Services, Ministry of Health and Family Welfare, Government of India.

3. Chakravarty S, Sen MK, Tyagi JS (2005) Diagnosis of extra pulmonary tuberculosis by smear, culture, and PCR using universal sample processing technology. J Clin Microbiol 43(9): 4357-4362.

4. Sharma SK, Mohan A (2004) Extra pulmonary tuberculosis. Indian J Med Res 120(4): 316-353.

5. Lange C, Mori T (2010) Advances in the diagnosis of tuberculosis. Respirology 15(2): 220-240.

6. Pai M, Ling DI (2008) Rapid diagnosis of extra pulmonary tuberculosis using nucleic acid amplification tests: what is the evidence? Future Microbiol 3(1): 1-4.

7. El Hajj HH, Marras SA, Tyagi S, Kramer FR, Alland D (2001) Detection of rifampicin resistance in Mycobacterium tuberculosis in a single tube with molecular beacons. J Clin Microbiol 39(11): 41314137.

8. Boehme CC, Nabeta P, Hillemann D, Nicol MP, Shennai $S$, et al. (2010) Rapid molecular detection of tuberculosis and Rifampin resistance. New Eng J Med 363(11): 1005-1015.

9. Piatek AS, Tyagi S, Pol AC, Telenti A, Miller LP, et al. (1998) Molecular beacon sequence analysis for detecting drug resistance in Mycobacterium tuberculosis. Nat Biotechnol 16(4): 359-363.

10. Blakemore R, Story E, Helb D, Kop J, Banada P, et al. (2010) Evaluation of the analytical performance of the Xpert MTB/ RIF assay. J Clin Microbiol 48(7): 2495-2501.

11. World Health Organization (2011) Automated realtime nucleic acid amplification technology for rapid and simultaneous detection of tuberculosis and rifampicin resistance: Xpert MTB/RIF system. Policy statement.

12. Pai M, Nathavitharana R (2014) Extrapulmonary tuberculosis: New diagnostics and new policies. Indian J Chest Dis Allied Sci 56: 71-73.

13. Clinical and Laboratory Standards Institute (2008) Laboratory detection and identification of Mycobacteria; Approved Guideline. Wayne.

14. Uria GA, Azcona JM, Midde M, Naik PK, Reddy S, et al. (2012) Rapid Diagnosis of Pulmonary and extrapulmonary tuberculosis in HIV-Infected Patients. Comparison of LED Fluorescent microscopy and the GeneXpert MTB/RIF assay in a District hospital in India. Tuberc Res Treat.

15. Lawn SD, Zumla AI (2012) Diagnosis of extrapulmonary tuberculosis using the Xpert MTB/RIF assay. Expert Rev Anti Infect Ther 10(6): 631-635.

16. Sajed AN, Khan S, Butt AS, Srwar A, Akhtar R, et al. (2014) Rapid detection of mycobacterium tuberculosis and Rifampicin resistance in extrapulmonary samples using GeneXpert MTB/RIF assay. IOSR J Den Med Sciences 13(11): 50-53.

17. Hillemann D, Rusch Gerdes S, Boehme C, Richter E (2011) Rapid Molecular Detection of Extrapulmonary Tuberculosis by the Automated GeneXpert MTB/RIF System. J Clin Microbiol 49(4): 1202-1205.

18. Causse M, Ruiz P, Gutiérrez Aroca J B, Casal M (2011) Comparison of two molecular methods for rapid diagnosis of extrapulmonary tuberculosis. J Clin Microbiol 49(8): 3065-3067.

19. Iqbal S, Ahmed R, Adhami SU, Mumtaz A (2011) Importance of polymerase chain reaction in diagnosis of pulmonary and extra-pulmonary tuberculosis. J Ayub Med Coll Abbottabad 23(1): 73-76.

20. Siddiqui MAM, Anuradha PR, Nagaman K, Vishnu PH (2013) Comparison of diagnostic modalities, BACTEC culture with polymerase chain reaction for diagnosis of extra pulmonary tuberculosis. J Med Allied Sci 3(2): 53-58.

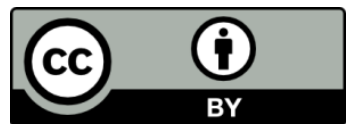

Pravin KN and Chourasia E. Use of GeneXpert Assay for Diagnosis of Tuberculosis From Body Fluid Specimens, a 2 Years Study . J Microbiol Copyright(C) Pravin KN and Chourasia E Biotechnol, 2016, 1(1): 000105. 